\title{
Encapsulation of Implicit and Explicit Memory in Sequence Learning
}

\author{
Paul J. Reber \\ UCSD School of Medicine
}

Larry R. Squire

Department of Veterans Affairs and UCSD School of Medicine

\begin{abstract}
Contrasts between implicit and explicit knowledge in the serial reaction time (SRT) paradigm have been challenged because they have depended on a single dissociation: intact implicit knowledge in the absence of corresponding explicit knowledge. In the SRT task, subjects respond with a corresponding keypress to a cue that appears in one of four locations. The cue follows a repeating sequence of locations, and subjects can exhibit knowledge of the repeating sequence through increasingly rapid performance (an implicit test) or by being able to recognize the sequence (an explicit test). In our
\end{abstract}

\section{INTRODUCTION}

Memory is not a single faculty but is composed of multiple separate abilities (Schacter, 1987; Squire, 1992; Tulving, 1985; Weiskrantz, 1990). One major distinction contrasts declarative (explicit) memory, which supports conscious memory of facts and events, with nondeclarative (implicit) memory, which supports a range of phenomena including habit learning, simple conditioning, and priming. The study of high-level cognition, in which task decisions are based on complex rules and sometimes detailed task information, has traditionally involved the assumption that task-relevant decision processes are guided mainly by declarative, conscious memory (Dulany, Carlson, \& Dewey, 1985; Perruchet \& Gallego, 1993; Shanks \& St. John, 1994a). However, a growing body of evidence has identified cognitive skill-learning tasks that depend primarily on nondeclarative memory (Squire \& Zola, 1996).

A powerful technique for examining the role of declarative and nondeclarative memory in cognitive skill learning is to study amnesic patients. Amnesic patients have damage to the medial temporal lobe or diencephalic structures and have severely impaired declarative memory. Yet, for a number of rather complex cognitive tasks, amnesic patients have been found to learn and retain information normally, despite being un- study, amnesic patients were given extensive SRT training. Their implicit and explicit test performance was compared to the performance of control subjects who memorized the training sequence. Compared with control subjects, amnesic patients exhibited superior performance on the implicit task and impaired performance on the explicit task. This crossover interaction suggests that implicit and explicit knowledge of the embedded sequence are separate and encapsulated and that they presumably depend on different brain systems.

able to recollect the task stimuli or the experimental context. These tasks include sequence learning (Nissen \& Bullemer, 1987; Reber \& Squire, 1994), prototype learning (Knowlton \& Squire, 1994), artificial grammar learning (Knowlton, Ramus, \& Squire, 1992), and probabilistic classification learning (Knowlton, Squire, \& Gluck, 1994). One challenge to this body of research in amnesic patients is that the work has depended on a single dissociation: intact implicit knowledge in the absence of corresponding explicit knowledge. This line of argument led to the proposal that there is a single, presumably explicit, source of knowledge and that implicit and explicit tests are simply differentially sensitive to detecting group differences (Shanks \& St. John, 1994a). This important, alternate idea has been termed the Sensitivity bypothesis.

In one relevant study, Reber and Squire (1994) compared the performance of amnesic patients and control subjects on the SRT task. In this task, a cue could appear in any one of four locations, and subjects responded as quickly as possible to each appearance of the cue by pressing the key directly beneath the cue. Subjects were not told that the cue followed a repeating sequence of locations, but with practice they nevertheless exhibited gradually decreasing reaction times to the cue. When the repeating sequence was replaced with a random sequence of locations, subjects exhibited slower reaction 
times, indicating that some of their previous speed was due to knowledge of the repeating sequence. The amount of sequence knowledge expressed in this way was equivalent for amnesic patients and control subjects. In contrast, the amnesic patients were markedly impaired relative to the control subjects at recognizing the repeating sequence when it was presented and, unlike the control subjects, they were unable to report the sequence verbally.

This finding demonstrates the standard single dissociation: intact implicit knowledge for the sequence and impaired explicit memory for the sequence. However, Shanks and St. John (1994a) pointed out that, if the implicit memory test were less sensitive to group differences than the explicit memory test, it could still be the case that a single source of knowledge supports performance on both tests. That is, the amnesic patients might have actually learned less about the sequence than control subjects, but only the explicit memory test is sensitive to this difference. The Sensitivity hypothesis would account for this pattern of results by supposing that the knowledge-performance relationship for the implicit memory test is such that subjects can express significant SRT skill with a relatively small amount of knowledge and that a small amount of knowledge is as effective as a large amount of knowledge at supporting performance. In addition, the explicit memory test may be relatively insensitive to a small amount of knowledge, but a large amount of knowledge is easily detectable.

By this hypothesis, amnesic patients should consistently perform slightly worse than control subjects on implicit memory tests (although the difference between groups need not be statistically significant). Consistent with this idea, in some of the studies carried out to date, overall performance of the amnesic patients was numerically worse than that of control subjects (see Shanks \& St. John, 1994b). Although such findings are consistent with the predictions of the single source of knowledge view and the Sensitivity hypothesis, they also do not contradict the multiple memory systems view. Perhaps control subjects can sometimes engage explicit memory strategies and thereby perform better than they could by relying solely on implicit memory. For this reason, it is not surprising that control subjects sometimes perform slightly, but not significantly, better than amnesic patients.

These alternate views cannot be easily distinguished on the basis of the evidence available from a single dissociation. One approach has been to study additional patient groups to try to establish the opposite dissociation (i.e., intact explicit memory in the presence of impaired implicit memory). Knowlton, Mangels, and Squire (1996) found that patients with Parkinson's disease were impaired at habit learning despite having intact explicit memory for the testing session. Another approach has been to examine the implicit learning of amnesic patients who are so severely impaired that they appear to have virtually no explicit memory at all. For example, a severely amnesic patient (EP) exhibited normal prototype learning (Squire \& Knowlton, 1995) and intact perceptual priming (Hamann \& Squire, 1997) but performed at chance on parallel tests of recognition memory.

An approach that has not been explored is to try to obtain a crossover interaction in the performance of amnesic patients and control subjects. If the multiple memory system view is correct, circumstances might be found such that one group exhibits significantly more implicit task knowledge than another group, but significantly less explicit task knowledge. Such a finding would contradict the Sensitivity hypothesis by demonstrating that the implicit and explicit memory tests are both sensitive enough to yield significant group differences. The approach taken in the current study was to try to create a situation in which amnesic patients had significantly more implicit knowledge than control subjects but significantly less explicit knowledge. We used the SRT task, which has previously been shown to support normal sequence learning in amnesic patients (Nissen \& Bullemer, 1987; Nissen, Willingham, \& Hartman, 1989; Reber \& Squire, 1994). The task has the additional advantage that tests of implicit sequence learning and explicit sequence memory necessarily depend on information about the embedded, repeating sequence itself. That is, the implicit and explicit memory tests depend on similar information.

Following training on the SRT task, knowledge of the repeating sequence was assessed with tests sensitive to either implicit or explicit knowledge. Explicit sequence knowledge was tested with a recognition memory test. Implicit sequence knowledge was tested by assessing SRT task performance. Specifically, learning the repeating sequence makes the location of the cue predictable and leads to faster keypresses (shorter reaction times) in response to successive appearances of the cue. Reaction times can also decrease due to the learning of general keypressing skills (nonspecific task learning). To assess the extent to which faster keypressing speed depends on specific knowledge of the training sequence, the training sequence was abruptly changed to a different, novel sequence without forewarning the subject. The amount of reaction-time slowing that occurred when the repeating sequence was changed indexes the amount of implicit knowledge that was acquired about the training sequence.

The important comparison was between amnesic patients who learned a repeating sequence implicitly by practicing it during extended training and four different groups of control subjects who learned the same sequence explicitly by observing and memorizing but without actually practicing the sequence. We hypothesized that amnesic patients, who have impaired declarative memory but are capable of normal sequence learning (Nissen \& Bullemer, 1987; Nissen, Willingham, \& 
Hartman, 1989; Reber \& Squire, 1994), would acquire significant implicit knowledge of the repeating sequence without acquiring much explicit knowledge about it. We further hypothesized that control subjects, who attempted to memorize the sequence without practicing it, would acquire explicit knowledge of the repeating sequence but would acquire little or no implicit knowledge about it.

\section{Nonspecific Learning}

As described above, implicit knowledge of the sequence was measured by noting the increase in reaction times that occurred between a block of trials containing the training sequence and an immediately following block of trials containing a novel sequence. However, there is a possible complication in measuring implicit knowledge in this way. If sufficient nonspecific learning occurred while practicing the training sequence, reaction times might be fast both during the final trials with the training sequence and also during subsequent trials with the novel sequence. In other words, evidence for specific knowledge of the sequence (namely, reaction time slowing during the novel sequence) could be masked if considerable nonspecific learning continued to occur during the test of implicit knowledge. Because of this possibility, a failure to find slower reaction times during the novel sequence could mean either that no implicit knowledge of the sequence had been acquired or that implicit knowledge had been acquired but was masked by ongoing nonspecific learning. We therefore gave pretraining on the SRT task to three groups of control subjects, who memorized the sequence. Pretraining permitted nonspecific learning to occur prior to the test of implicit knowledge and provided a way to demonstrate directly that nonspecific learning was not preventing the expression of specific sequence knowledge.

\section{Interference}

Subjects who receive pretraining on the SRT task will have acquired nonspecific task knowledge and will also have acquired implicit knowledge of the pretraining sequence itself. Another possible complication was that implicit knowledge of the pretraining sequence might interfere with learning and expressing implicit knowledge of the subsequent training sequence. To explore this possibility, one group of control subjects was given pretraining in which the cue appeared in a random series of locations. Another group received no pretraining so that there could be no interference from a previously learned sequence.

\section{RESULTS}

SRT performance was measured as mean reaction time (RT) for each 60-trial block of performance. In calculat- ing the mean RT for each 60-trial block, trials containing errors (i.e., an incorrect keystroke response by the subject) and trials containing RTs greater than $1000 \mathrm{msec}$ were eliminated. An average of $6 \%$ of the reaction times were eliminated.

\section{Pretraining}

Mean RTs for each block of pretraining are shown in

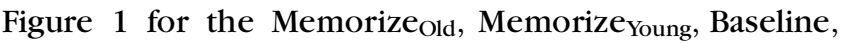
and Memorize Young Random Pretraining groups. For the Memorize $_{\text {Old }}$, Memorize $_{\text {Young, }}$ and Baseline groups, implicit sequence knowledge of the pretraining sequence $S_{2}$ was measured by the increase in reaction times on the fourth block, which contained five repetitions of a novel sequence $N_{2}$. Each group exhibited significant knowledge of the pretraining sequence $S_{2}$. The mean increase in RT on the fourth block for the Memorize Old group was $33.4 \pm 8.4 \mathrm{msec}, t(14)=3.97, p<0.01$. For the Memorize Young $_{\text {group, the mean increase in RT was }}$ $31.0 \pm 5.8 \mathrm{msec}, t(14)=5.40, p<0.001$. For the Baseline group, the mean increase in RT was $41.5 \pm 11.5 \mathrm{msec}$, $t(14)=3.60, p<0.01$.

Evidence of nonspecific SRT learning can also be seen in the pretraining performance. The final pretraining block for the Memorize ${ }_{\text {Old }}$ Memorize $_{\text {Young, }}$, and Baseline groups, which consisted of repetitions of a novel sequence, was generally performed faster than the first SRT pretraining block. The decrease in RT was $35.2 \pm 11.2$ msec for the Memorize old $_{\text {group, }}(14)=3.15, p<0.01$; $54.3 \pm 16.7 \mathrm{msec}$ for the Memorize $_{\text {Young }}$ group, $t(14)=$ $3.25, p<0.01$; and $17.9 \pm 8.4 \mathrm{msec}$ for the Baseline group, $t(14)=2.11, p<0.07$. In addition, the Mem-

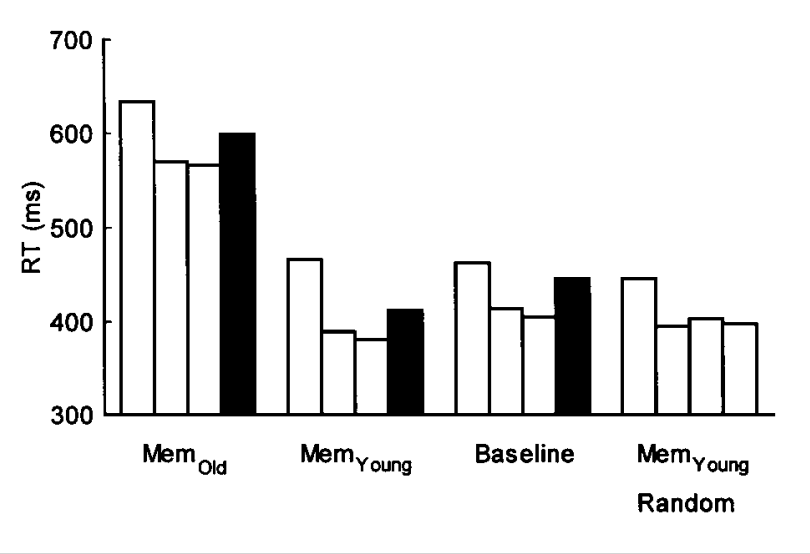

Figure 1. Average reaction time for each 60-trial block during pre-

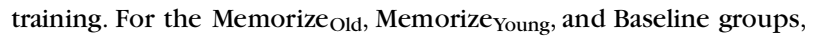
the open bars indicate the mean reaction time (RT) for the three 60trial blocks containing repetitions of sequence $S_{2}$, and the shaded bars indicate the mean RT for a 60-trial block containing repetitions of a novel sequence $N_{2}$. For the Memorizeyoung Random Pretraining group, each bar indicates the mean RT for 60-trial blocks in which the sequence of cue locations was pseudorandom. Standard errors for these scores ranged from 10 to $24 \mathrm{msec}$. 
orize $_{\text {Young }}$ Random Pretraining group also exhibited a decrease in mean RT of $48.4 \pm 9.4 \mathrm{msec}, F(1,9)=32.4$, $p<0.001$ that can only be attributed to nonspecific learning because the sequence of locations was unpredictable.

\section{Training}

Mean RTs for each block of SRT training for the amnesic patients and the Control (CON) group are shown in Figure 2. Due to computer error, the training data from the second training session were lost for two subjects in the CON group, and data were lost as well from the first eight blocks of the second session for one amnesic patient (EP). A $2 \times 2 \times 20$ analysis of variance (ANOVA) comparing the performance of amnesic patients and the CON group across the two sessions (20 training blocks each) yielded a significant effect of training block, $F(19$, 190) $=13.99, p<0.01$, which reflected the decreasing RTs during training. There was no effect of group or session and no interactions, $F \mathrm{~s}<1.10$. A second $2 \times 20$ ANOVA for the data from the first session yielded the same results.

\section{Verbal Report}

The verbal report score is the length of the maximum section of the repeating sequence contained in the subjects' responses (maximum $=12$, the length of the repeating sequence). The amnesic patients reported an average of $5.0( \pm 3.5)$ elements of the repeating sequence. The mean number of elements reported by the CON group was $4.2( \pm 0.3)$. For the groups who memorized the sequence, the mean number of elements con- tained in the responses was $4.7( \pm 0.5)$ for the Memorize Old group, $8.8( \pm 0.8)$ for the Memorize $_{\text {Young }}$ group, $9.0( \pm 0.9)$ for the Memorize ${ }_{\text {Young }}$ Random Pretraining group, and $8.7( \pm 0.8)$ for the Memorize Young No Pretraining group. Performance of the three Memorize $_{\text {Young }}$ groups (Memorize $_{\text {Young, }}$ Memorize Young Random Pretraining, and Memorize Young No Pretraining) was significantly better than the performance of the amnesic patients $(t \mathrm{~s}>2.71, p \mathrm{~s}<0.02)$ and better than the performance of the CON and Memorize ${ }_{\text {Old }}$ groups $(t \mathrm{~s}>4.33$, $p s<0.001)$. The performance of the CON and Memorize $_{\text {Old }}$ groups did not differ from that of the amnesic patients $(t \mathrm{~s}<0.50)$.

The important finding here was that the three Memorize $_{\text {young }}$ subject groups did learn most of the sequence (from 8.7 to 9.0 of the 12 sequence elements). The fact that the amnesic patients could report a fair part of the sequence suggests the possibility that verbal report performance was influenced by implicit knowledge (see "Discussion" for consideration of these ideas).

\section{Recognition}

The mean recognition score for each group is shown in Figure 3. The score for the amnesic patients reflects the average score for each of the two testing sessions. Table 1 shows the performance of individual patients. The CON, Memorize ${ }_{\text {Old }}$, Memorize $_{\text {Young, Memorize }}$ Young Random Pretraining, and Memorize Young No Pretraining groups all exhibited above-chance recognition of the repeating sequence $(t s>4.27, p s<0.002)$. The amnesic patients' recognition performance was not different from chance $(t<0.88)$. The amnesic patients were im-
Figure 2. Mean reaction time for each 60-trial block of repeating sequence $S_{1}$ across two training sessions. Filled squares $=5$ amnesic patients; open squares are 10 control subjects. Blocks 1 through 20 were administered on the first session, followed by the recognition memory test and the test of implicit knowledge (an additional 60 -trial block of the training sequence $S_{1}$ and a 60 trial block of a novel sequence $N_{1}$ ). Blocks 21 through 40 were administered 3 to 12 months later for the amnesic patients and 9 to 10 months later for the control subjects. Blocks 21 through 40 were also followed by tests of recognition memory and implicit knowledge.

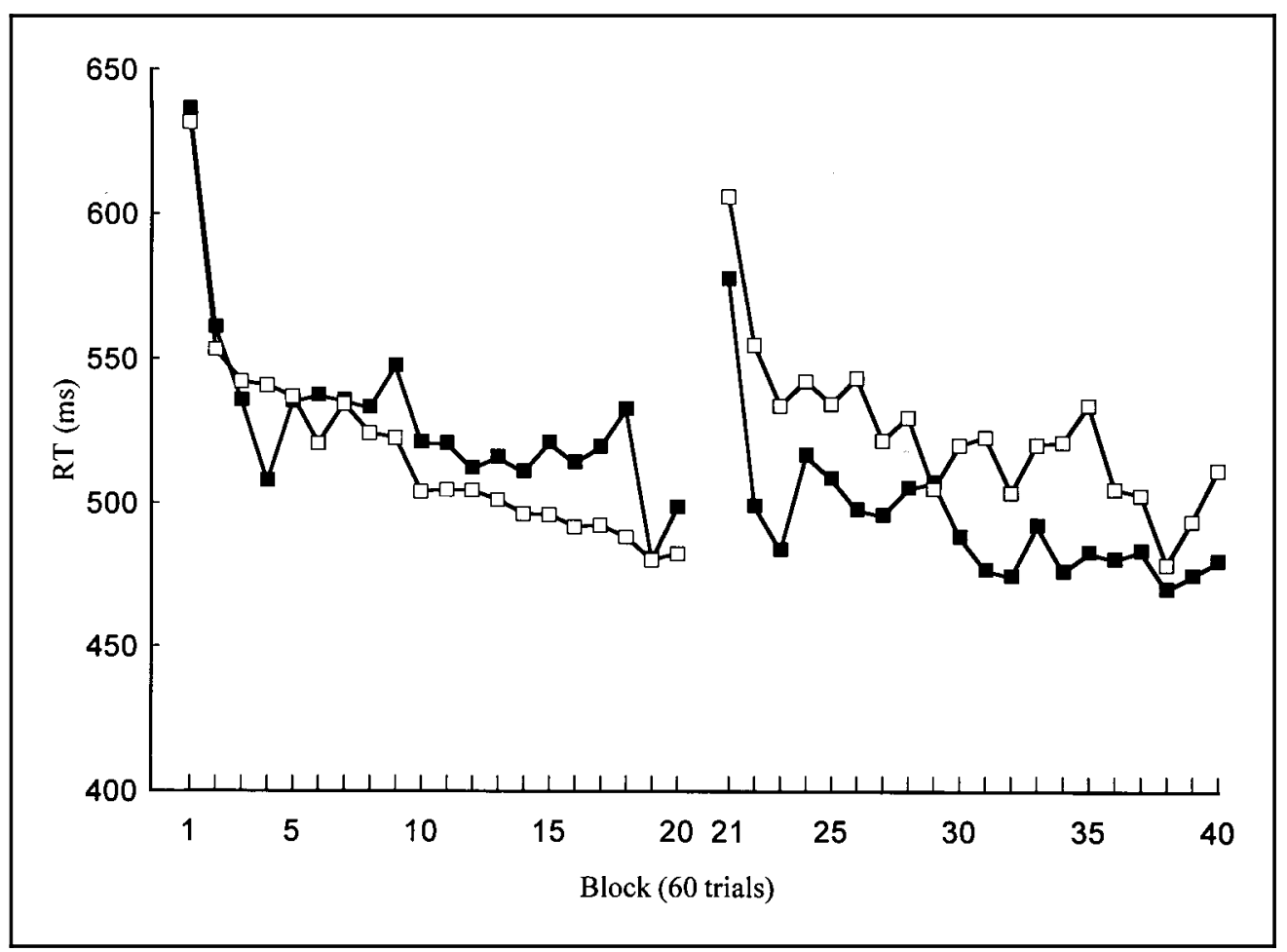


Figure 3. Recognition performance. In the recognition test, subjects rated five sequences (the target sequence and four foil sequences) on a 0 to 100 scale as to whether they had seen the sequence during training (either SRT practice or Sequence Memorization). Each subject was given a recognition score equal to the rating assigned to the target sequence minus the mean rating assigned to the other four sequences. Bars show group mean recognition scores. Brackets show the standard error of the group mean.

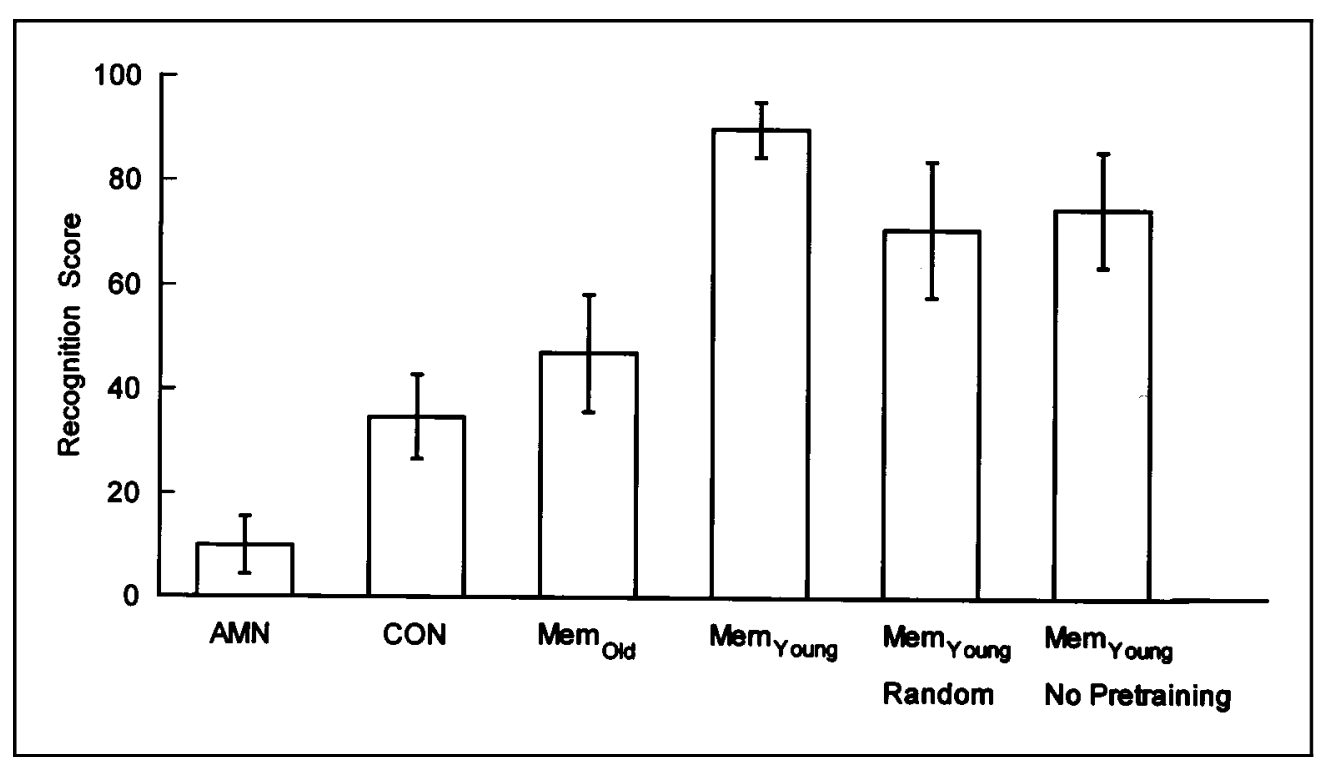

paired at recognizing the sequence compared with each of the control groups: CON group, $t(13)=2.58, p<0.03$, Memorize $_{\text {Old }}$ group, $t(18)=2.99, p<0.01$, Memorize Young group, $t(18)=8.12, p<0.001$, Memorize Young Random Pretraining group, $t(12)=4.67, p<0.001$, and Memorize $_{\text {Young }}$ No Pretraining group, $t(12)=4.91, p<$

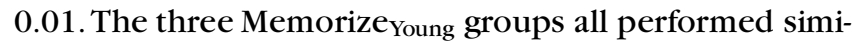
larly, $F(2,32)=0.93$. The CON and Memorize ${ }_{\text {Old }}$ groups also performed similarly, $t(23)=0.21$. The Memorize Young group remembered the sequence significantly better than the Memorize Old $_{\text {group, }} t(28)=3.47, p<0.01$. Finally, the CON group exhibited less recognition mem- ory of the repeating sequence than each of the three

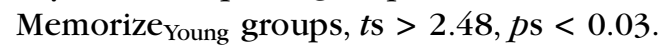

\section{Implicit Sequence Knowledge}

Performance on the implicit sequence knowledge test is shown in Figure 4 (see also Table 1 for the performance of individual amnesic patients in the two separate sessions). The amnesic patients exhibited significant knowledge of the repeating sequence as measured by a significant increase in reaction time on the second 60trial block (which contained repetitions of sequence $N_{1}$ ),

Table 1. Performance of Individual Amnesic Patients

\begin{tabular}{|c|c|c|c|c|c|c|c|c|c|}
\hline \multirow[b]{2}{*}{ Patient } & \multicolumn{3}{|c|}{ Session 1} & \multicolumn{3}{|c|}{ Session 2} & \multicolumn{3}{|c|}{ Both sessions } \\
\hline & Recog. & $\begin{array}{l}\text { Verbal } \\
\text { report }\end{array}$ & $\Delta R T$ & Recog. & $\begin{array}{l}\text { Verbal } \\
\text { report }\end{array}$ & $\Delta R T$ & Recog. & $\begin{array}{l}\text { Verbal } \\
\text { report }\end{array}$ & $\Delta R T$ \\
\hline PH & 31.2 & 8 & 7.3 & 12.5 & 4 & 48.2 & 21.9 & 6 & 27.8 \\
\hline $\mathrm{LJ}$ & 6.8 & 6 & -4.3 & -36.8 & 3 & 112.2 & 15.0 & 4.5 & 54.0 \\
\hline $\mathrm{NF}$ & 37.5 & 4 & 63.4 & 0 & 4 & -32.0 & 18.8 & 4 & 15.7 \\
\hline RC & 0 & 5 & 44.3 & -12.5 & 6 & 88.0 & -6.2 & 5.5 & 66.2 \\
\hline $\mathrm{EP}$ & 0 & 5 & 13.2 & 0 & 5 & 37.5 & 0 & 5 & 25.4 \\
\hline Means & 15.1 & 5.6 & 24.8 & -7.4 & 4.4 & 50.8 & 9.9 & 5.0 & 37.8 \\
\hline $\begin{array}{l}\text { Control means } \\
\quad(n=10)\end{array}$ & $\begin{array}{l}46.8 \\
(9.1)\end{array}$ & $\begin{array}{c}3.8 \\
(0.3)\end{array}$ & $\begin{array}{c}49.6 \\
(12.5)\end{array}$ & $\begin{array}{c}23.0 \\
(16.8)\end{array}$ & $\begin{array}{l}4.5 \\
(0.5)\end{array}$ & $\begin{array}{c}63.4 \\
(20.6)\end{array}$ & $\begin{array}{l}34.9 \\
(8.0)\end{array}$ & $\begin{array}{c}4.2 \\
(0.3)\end{array}$ & $\begin{array}{r}58.4 \\
(15.0)\end{array}$ \\
\hline
\end{tabular}

Note: Recog. indicates the scores of individual patients on the recognition test of explicit memory for the repeating sequence $\left(S_{1}\right)$. Verbal report indicates the length of the longest section of the repeating sequence contained in each patient's attempt to report the repeating sequence. $\Delta \mathrm{RT}$ indicates the difference in mean reaction time for the $S_{1}$ and $N_{1}$ (60-trial blocks) in the test of implicit knowledge for the repeating sequence. Each patient was tested twice in two separate sessions 3 to 5 months apart. Control subjects were tested twice in two separate sessions 9 to 10 months apart. For the control subjects, numbers in parentheses indicate the standard error. 
Figure 4. Reaction times (RT) on the Implicit Knowledge test. (A) Open bars indicate the mean RT for one 60-trial SRT block containing repetitions of the training sequence $S_{1}$. Shaded bars indicate the mean RT for the immediately following 60-trial SRT block, which contained repetitions of a novel sequence $N_{1}$. (B) Implicit knowledge for the training sequence $S_{1}$, as measured by the difference in mean RT during 60 trials of sequence $S_{1}$ and the immediately following 60 trials of sequence $N_{1}$. Thus the scores in panel (B) are subtraction scores based on the RTs in panel (A). Brackets show the standard error of the mean.
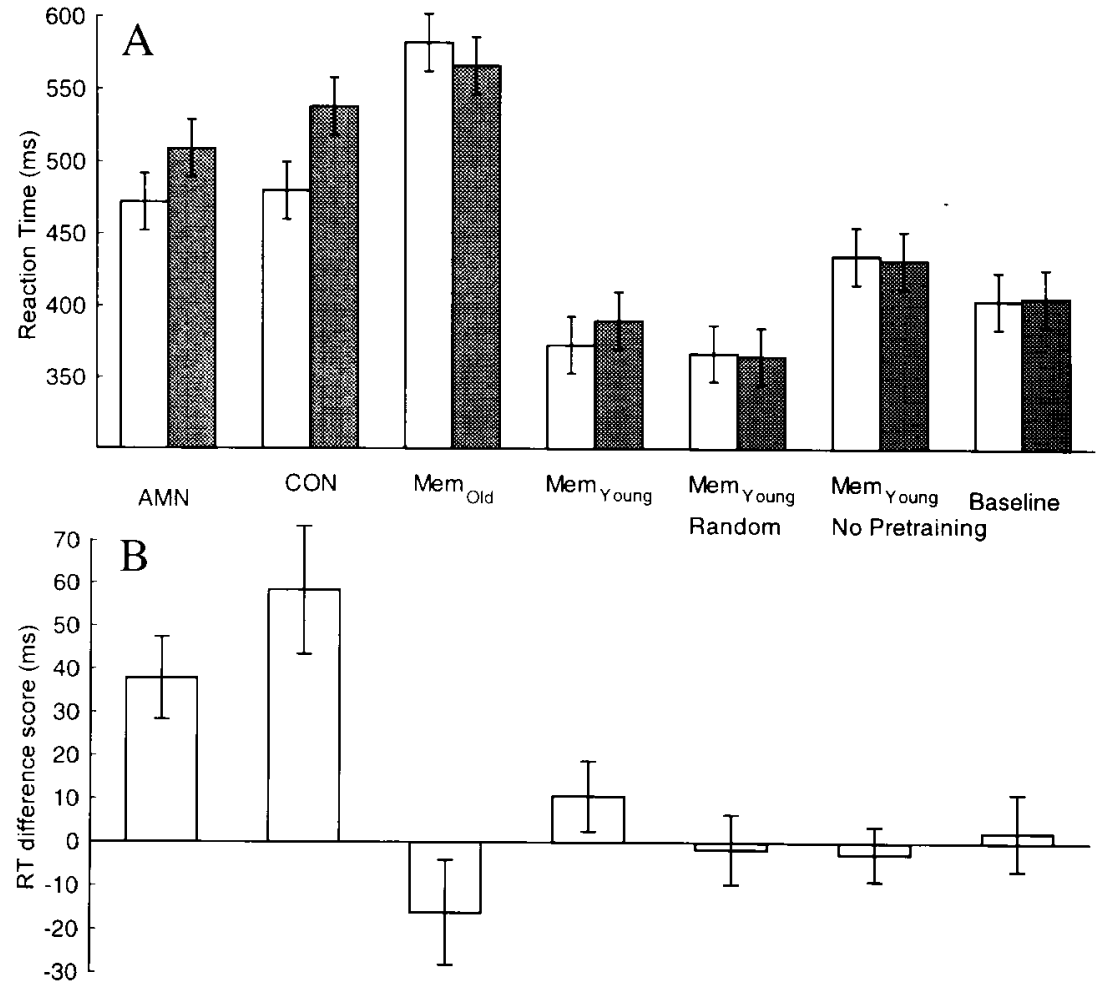

$t(4)=3.97, p<0.02$. The CON group also exhibited significant implicit sequence knowledge, $t(9)=3.90, p<$ 0.01 and performed similarly to the amnesic patients, $t(13)=1.16$. Note that the CON group did exhibit numerically greater RT slowdown than the amnesic patients as well as superior explicit knowledge (see Figure 4 and Table 1). This pattern of findings has been reported previously (Willingham, Nissen, \& Bullemer, 1989) and probably reflects a contribution of explicit sequence knowledge to SRT performance (see also "Discussion").

No other group exhibited significant knowledge of the repeating sequence $(t s<1.31)$. In addition, the amnesic patients expressed significantly more implicit knowledge of the sequence than the Memorize $_{\text {Old }}$ group, $t(18)=3.49, p<0.01$, the Memorize Young Random Pretraining group, $t(12)=2.99, p<0.02$, and the

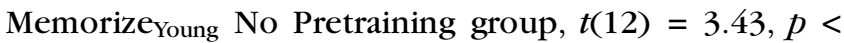
0.01 and marginally more implicit knowledge of the sequence than the Memorize ${ }_{\text {Young }}$ group, $t(18)=2.17, p$ $<0.055$. Likewise, the CON group exhibited more implicit sequence knowledge than the other three groups, $t \mathrm{~s}>3.04, p \mathrm{~s}<0.01$. There was a trend for the Memorize Young subjects to express more implicit sequence

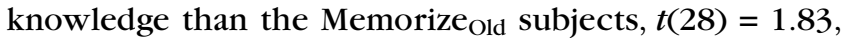
$p<0.08$. There was no difference in performance across the three Memorize Young groups, $F(2,32)=0.93$.

Finally, the Baseline group, which had not encountered the training sequence $\left(S_{1}\right)$ prior to the test of implicit sequence knowledge, also did not exhibit im- plicit knowledge. There was virtually no increase in RT on the second block of the test, $2.4 \pm 8.8 \mathrm{msec}, t(14)=$ 0.27 . The amount of sequence knowledge expressed by the Baseline group was less than that expressed by the amnesic patients, $t(18)=2.15, p<0.04$, and the CON group, $t(23)=3.43, p<0.01$. There was no difference in performance between the Baseline group and any of the four Memorize groups, $t \mathrm{~s}<1.23$.

\section{Crossover}

The performance scores of amnesic patients and control subjects on verbal report performance, the recognition memory test, and the implicit test of sequence knowledge were transformed to $z$ scores (using the mean and standard deviation for all subjects) in order to compare performance across tasks (Figure 5). The two explicit tests were averaged to produce a single $z$ score. Because there was no difference in performance on either of these tests for the three groups of younger subjects

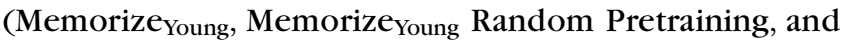

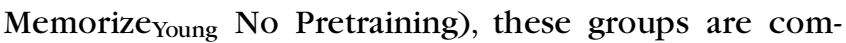
bined for this analysis. A repeated measures $2 \times 2$ ANOVA showed a significant interaction between group and task $F(1,38)=38.5, p<0.001$ with no effect of either group or task, $F \mathrm{~s}<1.20$. An ANOVA comparing the $z$ score data of the amnesic patients and the Memorize Old $_{\text {control }}$ subjects also shows a significant interaction between group and task, $F(1,18)=6.81, p<0.02$ with no reliable 


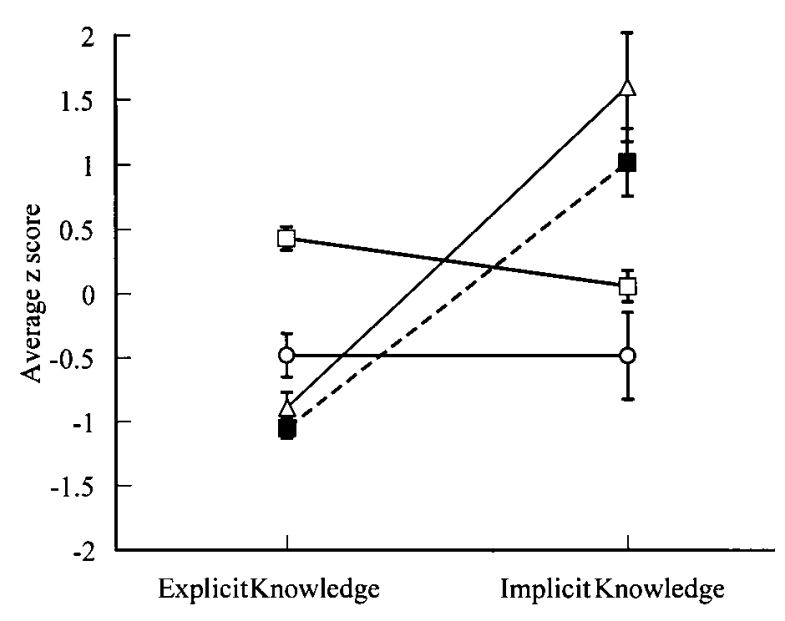

Figure 5. Crossover interaction between implicit and explicit knowledge. Filled squares indicate the performance of amnesic patients $(n=5)$. Open squares indicate the combined performance of the three groups of young subjects who tried to memorize the sequence (Memorize Young, Memorize Young Random Pretraining, and Memorize Young No Pretraining groups; $n=35$ ). Open circles indicate performance of Memorize old subjects $(n=15)$. Open triangles indicate the performance of the CON subjects who did not memorize but received extensive SRT training $(n=10)$. The score for explicit knowledge was derived from the verbal report score and the score on the recognition memory test (Figure 3). The score for implicit knowledge was the increase in mean reaction time obtained on the final 60-trial SRT block containing the novel sequence $N_{1}$ compared with the immediately preceding 60-trial SRT block containing the training sequence $S_{1}$. These scores were transformed to $z$ scores based on the means and standard deviations obtained for these two tests by all subjects $(n=65)$. For the explicit tests, $z$ scores for the verbal report and recognition memory test were averaged for each subject. Brackets indicate the standard error of the mean.

effects of group, $F(1,18)=2.99, p>0.10$ or task, $F(1$, 18) $=4.13, p>0.05$. In addition, the CON group shows a similar crossover interaction when compared to the three groups of younger subjects. A $2 \times 2$ ANOVA comparing the CON group to the combined performance of the three younger groups found a significant interaction between group and task, $F(1,43)=65.6, p<0.001$, no effect of group, $F(1,43)=0.31$, and a marginal effect of task $F(1,43)=3.29, p>0.07$. A similar ANOVA comparing the amnesic patients to the CON group found no effect of group, $F(1,13)=1.31, p>0.20$, an effect of task $F(1$, $13)=56.1, p<0.001$, and no interaction, $F(1,13)=0.38$. (Note that differences in task indicated by the ANOVAs cannot be readily interpreted because the scores for each task have been normalized to $z$ scores.)

\section{DISCUSSION}

The amnesic patients exhibited significantly more implicit sequence knowledge than any of the four control groups that memorized the sequence, and they simulta- neously exhibited significantly less explicit knowledge of the sequence than any of those four control groups. This crossover indicates that performance on the tests of implicit and explicit knowledge cannot depend on the same knowledge source. A crucial part of the crossover interaction is the fact that, after memorizing the sequence, the control subjects did not exhibit any implicit sequence knowledge. This result was observed in each of the four Memorize groups (Memorize ${ }_{\text {Old }}$, Memorize $_{\text {Young, }}$, Memorize Young Random Pretraining, and Memorize $_{\text {Young }}$ No Pretraining), which makes it possible to address several potentially complicating issues.

\section{Nonspecific Learning}

One possibility is that during the test of implicit sequence knowledge subjects acquired significant nonspecific knowledge of the SRT task that was not relevant to the repeating sequence. Significant nonspecific learning frequently occurs at the beginning of SRT practice. For example, it is apparent in Figure 1 that the RTs during the second 60-trial block of SRT training are faster than the RTs during the first block for SRT pretraining (for both repeating and random sequences). If significant nonspecific learning occurred during the implicit test of sequence knowledge, RTs for the $N_{1}$ block might be as fast or faster than RTs for the $S_{1}$ block (because the $N_{1}$ block followed the $S_{1}$ block), giving the appearance that no implicit knowledge had been acquired. The pretraining given to the Memorize ${ }_{\text {Old }}$ and Memorize Young groups addressed this possibility. The fact that the Memorize ${ }_{\text {Old }}$ and Memorize Young groups exhibited significant implicit knowledge of the pretraining sequence $S_{2}$ indicates that after three blocks of sequence learning, implicit sequence knowledge could be detected and that nonspecific SRT learning did not mask its expression. Accordingly, the implicit test of sequence knowledge for the training sequence $S_{1}$, which was administered shortly afterward, could not have been contaminated by nonspecific SRT learning for these groups.

\section{Interference}

It seemed possible that, in the case of the Memorize Old and Memorize Young groups, pretraining might interfere with the learning or expression of the second training sequence $\left(S_{1}\right)$. If so, the absence of measurable implicit knowledge in these groups might be due to interference caused by pretraining rather than the failure to acquire implicit knowledge. However, this possibility can be discounted. The Memorize Young Random Pretraining group exhibited no implicit knowledge of sequence $S_{1}$ after having received pretraining with a random sequence,

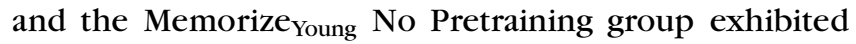
no implicit sequence knowledge when no pretraining occurred. 


\section{Baseline Differences in RT}

A final concern was that the younger control groups exhibited much faster RTs overall than both the amnesic patients and the older control groups (CON and Memorize $_{\text {Old }}$ groups). Accordingly, it seemed possible that the younger control groups might not demonstrate implicit sequence knowledge because they were faster at responding and relatively insusceptible to RT slowing when the novel sequence was introduced. However, the Memorize $_{\text {old }}$ group performed similarly to the amnesic patients in overall RT during the implicit sequence knowledge test (see Figure 4A) but, like the three younger control groups, exhibited no implicit sequence knowledge. In addition, the fact that the Memorize Young group and the Baseline group exhibited significant sequence knowledge during pretraining suggests that the sensitivity of the implicit sequence knowledge test is not being compromised by generally fast RTs in the case of younger control subjects.

\section{Key Findings}

In summary, although these potential complications all seemed plausible a priori, none appears to have been a significant factor. The four Memorize groups all behaved similarly to each other on the implicit knowledge test for sequence $S_{1}$. All four groups also performed similarly to the subjects in the Baseline group, who had no experience with the training sequence prior to the test of implicit sequence knowledge. Thus, neither pretraining, interference, or baseline differences in RT had a significant impact on performance on the implicit knowledge test. Together, these four control groups address several possible concerns and strengthen the finding that explicit memorization training does not improve performance on the implicit sequence knowledge test.

To establish a crossover interaction between explicit and implicit memory, it is important to ensure that explicit memorization training did lead to significant explicit knowledge of the repeating sequence. Although the performance of the four Memorize groups on the recognition memory test could conceivably have arisen from explicit knowledge of only the first few sequence elements (just enough to distinguish the target sequence from the foils), the fact that the three Memorize groups reported the majority of the sequence (mean = 8.7 to 9.0 elements out of 12 possible elements) indicates directly that these subjects did acquire significant explicit knowledge. In spite of this explicit knowledge, these subjects exhibited little implicit knowledge of the sequence on the subsequent SRT test. Thus, as the crossover interaction illustrates, explicit memorization training of a sequence led to explicit knowledge of the sequence without corresponding implicit knowledge.

It is interesting to note that, although explicit memorization training appears to lead to significant explicit knowledge of the training sequence, the CON and Memorize $_{\text {Old }}$ groups did not exhibit better performance in their verbal report than the amnesic patients did (although these two control groups did perform significantly better on the recognition test). It seems likely that the verbal report scores obtained by the amnesic patients were influenced by their implicit sequence knowledge. For example, in several instances after training, some amnesic patients denied that there was any embedded repeating sequence but after repeated encouragement to guess, they reported (typically by pointing) a fair number of sequence elements (e.g., PH, session 1, Verbal report score $=8$; EP, for both sessions, Verbal report score $=5$ ). Notably, patient EP is so severely amnesic that he has no detectable declarative memory capacity (Hamann \& Squire, 1997).

It might seem surprising that subjects can acquire explicit sequence knowledge but then not express this knowledge on an implicit memory test for the same sequence. Indeed, several studies have reported that explicit knowledge of a repeating sequence can contribute to performance on the SRT task. In one study (Willingham, Nissen, \& Bullemer, 1989), subjects who achieved full explicit knowledge of a repeating sequence while performing the SRT task exhibited significantly faster RTs than subjects who did not achieve full explicit knowledge of the sequence. This finding suggests that explicit sequence knowledge can contribute to performance on the SRT task. Similarly, Frensch and Miner (1994, Experiment 1) found that subjects who were given explicit instructions to attempt to discover the embedded repeating sequence while performing the SRT task showed better sequence learning than did subjects who were not so instructed. An important difference between these studies and ours is that the subjects in both the Willingham et al. and Frensch and Miner (1994) studies acquired their explicit knowledge while practicing the SRT task. In contrast, our control subjects had no practice on the SRT task with the training sequence before receiving the implicit knowledge test. We suggest that explicit sequence knowledge can improve SRT performance only after some implicit knowledge of that sequence has been acquired.

In another relevant study, Curran and Keele (1993, Experiment 1) explicitly told subjects the sequence of locations the cue would follow before beginning SRT training. These subjects appeared to exhibit faster reaction times on the first block of 120 SRT trials than subjects who had not been explicitly instructed (statistics for this comparison are not reported). However, there were several key differences between our procedure and the procedure used by Curran and Keele. In the Curran and Keele experiment, the embedded sequence was only six locations in length, making it much easier to learn compared with our twelve-location sequence. Also, the first reported RT means are derived from 120-trial blocks of SRT performance. In our test of 
implicit knowledge, subjects received only 60 trials of practice with the training sequence followed by 60 trials of a novel sequence. This point deserves emphasis because during 120 trials of SRT practice with a six-item sequence, it is likely that significant implicit sequence learning occurred. Thus, the apparently faster RTs in the first SRT block may have been supported by the explicit sequence knowledge as well as by implicit sequence knowledge that was acquired during the first block of SRT practice.

Howard, Mutter, and Howard (1992) also report a condition under which subjects appeared to be able to express implicit knowledge of a repeating sequence without prior SRT training. In this study, subjects who simply observed a repeating sequence subsequently exhibited as much sequence knowledge on an implicit test as subjects who had actually practiced the SRT task with the training sequence. Although these subjects were not explicitly told to memorize the sequence (as our subjects were), there is some reason to suspect that their subjects may have acquired explicit knowledge of the repeating sequence during their extended observation period. In the Howard et al. experiment, subjects observed 30 repetitions of the sequence before starting to make keypress responses. In addition, the sequence used in the Howard et al. experiment was only 10 locations in length. By contrast, our subjects exhibited significant explicit sequence knowledge after observing a 12-item sequence only five times (although our subjects were instructed to attempt to memorize the sequence). We suggest that the subjects in the Howard et al. experiment had ample time to develop some explicit knowledge of the sequence. The test of implicit sequence knowledge used by Howard et al. gave subjects 100 trials of practice with the training sequence, followed by 100 trials of a random sequence. Significant learning of the simpler 10-location sequence may have occurred during the 100 trials of practice with the training sequence. Figure 5 of Howard et al. supports this idea, showing that during the 100 trials of SRT practice that preceded the switch to a random sequence, response times improved substantially. Thus, once again, good performance on their test of implicit sequence knowledge may have been supported by implicit knowledge acquired during the 100 trials of SRT practice, together with the explicit sequence knowledge acquired during observation. In each of these studies (Curran \& Keele, 1993; Frensch \& Miner, 1994; Howard et al. 1992; Willingham et al. 1989), it appears to be the conjunction of explicit sequence knowledge and some implicit sequence knowledge that enabled subjects to exhibit very rapid response times during the SRT task.

It is worth noting that the CON group in our study, who received extensive SRT practice and also acquired some explicit sequence knowledge, exhibited a numerically larger RT slowdown on the implicit sequence test than the amnesic patients (although this numerical dif- ference, $38 \mathrm{msec}$ vs. $58 \mathrm{msec}$, was not significant). If this numerical difference were reliable, it could mean that the CON group was able to apply some explicit knowledge to the implicit memory test as the result of their SRT practice, as in Curran and Keele (1993); Frensch and Miner (1994); Howard et al. (1992); and Willingham et al. (1989). In addition, if explicit knowledge can contribute to SRT performance when subjects have also acquired sufficient implicit sequence knowledge, control subjects could exhibit numerically (but not necessarily reliably) better SRT performance than amnesic patients (e.g., Reber \& Squire, 1994).

Previous research indicated that implicit learning of a repeating sequence in the SRT paradigm is intact in amnesic patients (Nissen \& Bullemer, 1987; Nissen, Willingham, \& Hartman, 1989; Reber \& Squire, 1994). This single dissociation suggested that implicit sequence learning does not depend on declarative memory (which is impaired in amnesic patients), but it did not address the Sensitivity hypothesis that there is a single memory system and that the implicit and explicit tests are simply differentially sensitive to group differences (Shanks \& St. John 1994a). The Sensitivity hypothesis predicts that control subjects should typically perform numerically better than amnesic patients, and it therefore provides an alternate account of the single dissociations reported here and elsewhere (Nissen \& Bullemer, 1987; Reber \& Squire, 1994), as well as studies indicating that SRT performance can depend on both implicit and explicit sequence knowledge (Willingham et al. 1989; Howard et al. 1992). However, the Sensitivity hypothesis cannot account for the crossover interaction reported in the current study. We suggest that the crossover interaction observed in our study is driven by the encapsulation of explicit and implicit sequence knowledge in separate memory systems.

It is notable that the crossover interaction between implicit and explicit knowledge can be described as resulting from transfer-appropriate processing (Morris, Bransford, \& Franks, 1977; Roediger \& Blaxton, 1987; Roediger, 1990). The best performance on the tests of implicit and explicit sequence knowledge followed training (practice or memorization) that was appropriate for each test. One could then suppose that transfer-appropriate processing explains the results because the contexts for the two tests are so different that transfer between the two tasks is quite limited. However, a transfer-appropriate processing account of performance is also consistent with the multiple memory systems view (Schacter, 1990). The idea is that the separate brain regions supporting parallel learning and memory systems operate according to the transfer-appropriate processing principle. We suppose that an explicit representation afforded by memorization training is supported by the medial temporal lobe structures that support declarative memory. An implicit representation of sequence knowledge afforded by SRT practice is supported by different 
brain areas (e.g., the neostriatum, supplementary motor area, and motor cortex, see below). The key point for understanding the crossover interaction is that it is more difficult to apply explicit knowledge to an implicit test (or vice versa) than to perform in conditions where the type of test matches the training that was given. In our experiment, control subjects who memorized the sequence developed an explicit representation of the repeating sequence that supported a high level of performance on the recognition memory test but did not support expression of sequence knowledge when this information was transferred to the implicit test. By contrast, the amnesic patients developed an implicit representation of the repeating sequence through SRT practice without developing explicit sequence knowledge (due to their declarative memory impairment). In the case of the patients, implicit representation of sequence knowledge supported superior performance on the implicit test but did not contribute to performance on the recognition memory test. The resulting crossover interaction suggests that there are separate representations of sequence knowledge (in a single memory system) that support performance on both implicit and explicit tests.

It should be clear that the conclusion that there are separate underlying memory systems for implicit and explicit sequence knowledge depends not only on the finding of a crossover dissociation presented here but also on previous reports of preserved learning on this task by amnesic patients (Nissen et al. 1989; Nissen \& Bullemer, 1987; Reber \& Squire, 1994). The fact that amnesic patients are selectively impaired at explicit sequence learning suggests that this type of learning depends on the medial temporal lobe and that implicit sequence knowledge is supported by brain areas outside the medial temporal lobe. As has been noted, evaluating the performance of amnesic patients in isolation has not ruled out the possibility that observed single dissociations arise from sensitivity differences between tests of implicit and explicit knowledge (e.g., Shanks \& St. John 1994a). The crossover interaction demonstrated here indicates that differential performance on implicit and explicit tests does not simply arise from sensitivity differences. The combination of two results (selective impairment in amnesic patients and a crossover interaction) indicates that implicit and explicit sequence learning must depend on separate brain systems supporting separate representations of sequence knowledge.

A number of reports have provided converging evidence that SRT performance and implicit sequence learning are at least partially supported by the neostriatal habit learning system. Patients with neostriatal damage due to either Huntington's disease (HD) or Parkinson's disease (PD) were impaired on learning the SRT task (for HD patients, Knopman \& Nissen, 1991; Willingham \& Koroshetz, 1993; for PD patients, Jackson, Jackson, Harrison, Henderson \& Kennard, 1995; Pascual-Leone et al.,
1994). Neuroimaging with positron emission tomography indicated that the sensorimotor cortex and neostriatum were active during SRT learning in conditions in which little explicit knowledge was acquired (Grafton, Hazeltine, \& Ivry, 1995; Rauch et al., 1995). Both studies also found evidence for the involvement of different structures when the sequence was learned explicitly. In a study of motor cortex mapping using transcranial magnetic stimulation (TMS) at several points during extended SRT practice (Pascual-Leone, Grafman, \& Hallett, 1994), the extent of the cortical map and the muscle response amplitude to TMS increased as subjects developed implicit knowledge of the repeating sequence. Thus, the findings from all these studies are consistent with the involvement of a corticostriatal system during implicit learning of the SRT task.

In this view, amnesic patients can demonstrate normal sequence learning because their motor cortex and neostriatum are intact. CON subjects can also learn the sequence implicitly through SRT practice, and in addition, by virtue of their intact medial temporal lobe and diencephalic structures, they are able to memorize a sequence of cue locations. However, explicit sequence knowledge is encapsulated and does not contribute to SRT performance, at least not during the early stages of practice. With continuing practice, control subjects may be able to improve their performance by engaging in declarative memory strategies (Willingham et al., 1989). The encapsulation of declarative and nondeclarative memory is also suggested by our earlier report (Reber \& Squire, 1994). In that study, the control subjects acquired more explicit knowledge of the sequence during SRT practice than the amnesic patients. However, both groups performed similarly on the SRT task of implicit memory. That is, the explicit memory acquired by control subjects did not contribute to performance on the implicit test.

It is important to note that the independence of implicit and explicit memory in the SRT task demonstrated here does not imply that implicit and explicit sequence knowledge cannot interact. The proposal that implicit and explicit memory in the SRT task are encapsulated refers to the operation of separate neural substrates that support the two types of memory. Yet, in many paradigms, behavior can reflect the operation of both systems. Thus, behavior arises from the operation of two memory systems, each of which provides a different kind of information, and both sources of information contribute to overall performance.

The idea that implicit sequence learning is supported by a corticostriatal circuit that is functionally distinct from the brain system supporting declarative memory is now based on a number of different studies involving behavioral data from normal subjects, behavioral data from several neurological patient groups, and functional neuroimaging data. The preponderance of evidence points to the operation of functionally and neuroana- 
tomically distinct memory systems that can operate in parallel during high-level cognitive tasks.

\section{METHOD}

\section{Subjects}

\section{Amnesic Patients}

Five amnesic patients (four men and one woman) participated in this study. Two of the patients have alcoholic Korsakoff's syndrome. Both have participated in quantitative magnetic resonance imaging (MRI) studies that demonstrated reductions in the volume of the mammillary nuclei (for RC, Squire, Amaral, \& Press 1990; for NF, unpublished observations). Patient NF also has bilateral reduction in the size of the hippocampal formation. The remaining three patients have bilateral hippocampal formation damage (for PH, Polich \& Squire, 1993; for EP, Squire \& Knowlton, 1995; for LJ, unpublished observations). Patient PH had a history of 1- to 2-min attacks (of possible epileptic origin) in association with gastric symptoms and transient memory impairment. In 1989 he suffered a series of small attacks that resulted in marked and persisting memory impairment. Patient EP developed profound anterograde and retrograde amnesia in 1992 after herpes simplex encephalitis. Patient LJ became amnesic gradually in 1988 to 1989 without any known precipitating event. Her memory impairment has remained stable since that time.

The patients averaged 69 years of age at the beginning of the study and had an average of 12.8 years of education. Immediate and delayed $(12 \mathrm{~min})$ prose recall averaged 3.2 and 0.0 segments, respectively (Gilbert, Levee, \& Catalano, 1968; maximum score $=21$ ). Scores on other memory tests appear in Tables 2 and 3. The mean score on the Dementia Rating Scale (DRS) was 127.6 (Mattis, 1976; maximum score $=144$ ). Most of the points lost on the DRS were from the memory subportion of the test (mean points lost $=10.6$ ). The mean score for the Boston Naming Test was 53.4 (Kaplan, Goodglass, \& Weintraub, 1983 ; maximum score $=60$ ). Scores for healthy subjects on these tests can be found elsewhere Janowsky, Shimamura, \& Squire, 1989; Squire et al. 1990).

\section{CON Subjects}

The control subjects (26 men and 44 women) were either employees or volunteers at the San Diego Veterans Affairs Medical Center or were recruited from the retirement community of the University of California at San Diego. The control subjects were assigned to six separate groups (Table 3). Two of the control subject groups (CON, $n=10$; Memorize $_{\text {Old }}, n=15$ ) were matched to the amnesic patients with respect to the mean and range of their ages, years of education, and scores on the Information and Vocabulary subtests of the Wechsler Adult Intelligence Scale-Revised (WAIS-R). These two groups averaged 66.6 years of age, 14.2 years of education, and 20.5 and 52.8 on the Information and Vocabulary subtests, respectively (amnesic patients $=19.7$ and 51.3, respectively). Immediate and delayed prose recall averaged 7.5 and 6.2 segments, respectively.

Four groups of younger control subjects were also tested (Table 4): Memorize Young, $(n=15)$ Memorize $_{\text {Young }}$

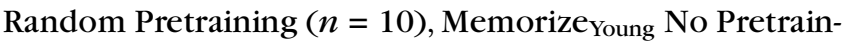
ing $(n=10)$, and Baseline $(n=10)$. These four groups averaged 26.2 years of age (range of group means $=25.2$ to 27.1 years) and 16.8 years of education (range of group means $=16.4$ to 17.3 years).

Table 2. Characteristics of Amnesic Patients

$W M S-R$

\begin{tabular}{|c|c|c|c|c|c|c|c|c|}
\hline \multirow[b]{2}{*}{ Patient } & \multirow[b]{2}{*}{ Lesion } & \multirow[b]{2}{*}{$\begin{array}{c}\text { Age } \\
\text { (years) }\end{array}$} & \multirow[b]{2}{*}{$\begin{array}{c}\text { WAIS-R } \\
I Q\end{array}$} & & & & & \\
\hline & & & & Attention & Verbal & Visual & General & Delay \\
\hline PH & $\mathrm{HF}$ & 74 & 120 & 117 & 67 & 83 & 70 & 57 \\
\hline $\mathrm{LJ}$ & $\mathrm{HF}$ & 58 & 98 & 105 & 83 & 60 & 69 & $<50$ \\
\hline $\mathrm{RC}^{a}$ & Dien & 79 & 106 & 115 & 76 & 97 & 80 & 72 \\
\hline $\mathrm{NF}^{a}$ & Dien & 60 & 94 & 91 & 62 & 73 & 53 & $<50$ \\
\hline EP & $\mathrm{HF}$ & 74 & 103 & 94 & 57 & 82 & 61 & 56 \\
\hline Means & & 69 & 104.2 & 104.4 & 69 & 79 & 66.7 & 57 \\
\hline
\end{tabular}

Note: WAIS-R = Wechsler Adult Intelligence Scale-Revised; WMS-R = Wechsler Memory Scale-Revised. HF = Hippocampal formation; Dien = Diencephalon. The WAIS-R and the WMS-R indices yield a mean score of 100 in the normal population with a standard deviation of 15 . The WMS-R does not provide scores for subjects who score below 50. Therefore, the two scores below 50 were scored as 50 for calculating a group mean.

$a$ These patients have alcoholic Korsakoff's syndrome. NF has reduced volume of the hippocampal formation in addition to a diencephalic lesion. 
Table 3. Memory Test Performance

\begin{tabular}{|c|c|c|c|c|c|c|c|c|}
\hline \multirow[t]{2}{*}{ Patient } & \multirow{2}{*}{$\begin{array}{c}\begin{array}{c}\text { Diagram } \\
\text { recall }\end{array} \\
3\end{array}$} & \multicolumn{3}{|c|}{ Paired associates } & \multirow{2}{*}{$\begin{array}{c}\begin{array}{c}\text { Word } \\
\text { recall }(\%)\end{array} \\
27\end{array}$} & \multirow{2}{*}{$\begin{array}{c}\begin{array}{c}\text { Word } \\
\text { recognition }(\%)\end{array} \\
84\end{array}$} & \multirow{2}{*}{$\begin{array}{c}50 \\
\text { words }\end{array}$} & \multirow{2}{*}{$\begin{array}{c}50 \\
\text { faces }\end{array}$} \\
\hline & & 0 & 0 & 1 & & & & \\
\hline LJ & 3 & 0 & 0 & 0 & 40 & 93 & 33 & 29 \\
\hline $\mathrm{RC}$ & 3 & 0 & 0 & 3 & 19 & 85 & 37 & 30 \\
\hline NF & 4 & 0 & 0 & 2 & 36 & 76 & 28 & 27 \\
\hline EP & 0 & 0 & 0 & 0 & 24 & 65 & 24 & 28 \\
\hline Means & 2.6 & 0 & 0 & 1.2 & 29.2 & 80.6 & 31.6 & 29.6 \\
\hline $\begin{array}{l}\text { Control means } \\
\quad(n=8)\end{array}$ & 20.6 & 6.0 & 7.6 & 8.9 & 71.0 & 97.0 & 41.1 & 38.1 \\
\hline
\end{tabular}

Note: The diagram recall score is based on delayed $(12-\mathrm{min})$ reproduction of the Rey-Osterrieth figure (Osterrieth, 1944; maximum score $=36$ ). The average score for the amnesic patients for copying the figure was 27.6, a normal score (Kritchevsky et al. 1988). The paired associate scores are the number of word pairs recalled on three successive trials (maximum score $=10$ per trial). The word recall score is the percentage of words recalled across five successive study-test trials. The word recognition score is the percentage of words identified correctly by yes/no recognition across five consecutive study-test trials. The score for words and faces is based on a 24 -hr delayed recognition test of 50 words or 50 faces (modified from Warrington, 1984; maximum score $=50$, chance $=25$ ). The mean scores for control subjects shown for these tests are from Squire and Shimamura (1986).

\section{Materials}

\section{The SRT Task}

The SRT task was presented on an EPSON 650 color laptop computer (10.4-in dual-scan LCD screen). Four dashes $(0.5 \mathrm{~cm}$ in width and $4.0 \mathrm{~cm}$ apart) appeared continuously $1.0 \mathrm{~cm}$ from the bottom of the screen to denote the four possible locations of the cue. The cue was an asterisk $0.4 \mathrm{~cm}$ wide that could appear $5.3 \mathrm{~cm}$ above any one of the four dashes. Subjects were in- structed, "When the asterisk appears, press the key underneath it as quickly as you can." Responses were made on the computer keyboard using the four keys directly beneath the dashes: $\mathrm{c}, \mathrm{b}, \mathrm{m}$, and the period key. These keys were marked with white stickers to indicate where the subjects should place their fingers during the task. Subjects used two fingers from each hand, usually the first and second digits, and were instructed to maintain contact with the four marked keys throughout training. A correct keypress caused the asterisk to disappear and

Table 4. Experimental Procedure

\begin{tabular}{|c|c|c|c|c|}
\hline Group & Pretraining & Training & Explicit test & $\begin{array}{l}\text { Implicit } \\
\text { test }\end{array}$ \\
\hline Amnesic patients $(n=5)$ & - & Practice 20 blocks $S_{1}$ & Verbal report, recognize $S_{1}$ & $S_{1} N_{1}$ \\
\hline Control Subjects $(n=10)$ & - & Practice 20 blocks $S_{1}$ & Verbal report, recognize $S_{1}$ & $S_{1} N_{1}$ \\
\hline $\operatorname{Memorize}_{\text {Old }}(n=15)$ & $S_{2} S_{2} S_{2} N_{2}$ & Memorize 1 block $S_{1}$ & Verbal report, recognize $S_{1}$ & $S_{1} N_{1}$ \\
\hline Memorize Young $_{(n=15)}$ & $S_{2} S_{2} S_{2} N_{2}$ & Memorize 1 block $S_{1}$ & Verbal report, recognize $S_{1}$ & $S_{1} N_{1}$ \\
\hline $\begin{array}{l}\text { Memorize }{ }_{\text {Young Random Pretraining }} \\
\quad(n=10)\end{array}$ & $R_{1} R_{2} R_{3} R_{4}$ & Memorize 1 block $S_{1}$ & Verbal report, recognize $S_{1}$ & $S_{1} N_{1}$ \\
\hline $\begin{array}{l}\text { Memorize }{ }_{\text {Young No Pretraining }} \\
\quad(n=10)\end{array}$ & - & Memorize 1 block $S_{1}$ & Verbal report, recognize $S_{1}$ & $S_{1} N_{1}$ \\
\hline Baseline $(n=15)$ & $S_{2} S_{2} S_{2} N_{2}$ & - & - & $S_{1} N_{1}$ \\
\hline
\end{tabular}

Note: $S_{1}$ represents one 60-trial block of the training sequence (124313214234). $N_{1}$ represents one 60-trial block of a novel sequence (343213142412). $S_{2}$ represents one 60-trial block of a second pretraining sequence (232413143421). $N_{2}$ represents one 60-trial block of a second novel sequence (432423121413). Each 60-trial block contains five repetitions of the sequence. $R_{1-4}$ represent four 60 -trial blocks of a balanced, random sequence of cue locations (see text). The amnesic patients were tested twice with the procedure shown, with an interval of 3 to 5 months between tests. The control subjects were also tested twice, with an interval of 9 to 10 months between tests. The four groups of control subjects who memorized the repeating sequence are grouped together by horizontal lines. These groups varied either in mean age or the type of SRT pretraining they received before attempting to memorize the repeating sequence. 
then reappear in a new location after a 250 -msec delay. The cue never appeared in the same location on successive trials. An incorrect response resulted in the computer beeping once, and the next trial started only after a correct response had been made. The task was administered in 60-trial blocks with a 15-sec break following each block.

\section{Repeating Sequences and Random Sequences}

Each 60-trial block of the SRT task contained five repetitions of a 12-location sequence (except as noted below for pretraining of the Memorize Young Random Pretraining group). Each 12-location sequence contained three occurrences of each of the 4 possible cue locations and one occurrence of each of the 12 possible transitions between locations (e.g., 12, 13, 14, 21, 23, etc.). Thus, each sequence was balanced (or "ambiguous"; Curran \& Keele, 1993). With a balanced sequence, subjects cannot predict the next location of the cue simply by learning location or transition frequencies. They must learn second-order conditional (SOC) associations (Reed \& Johnson, 1994). That is, in order to predict the next cue location, they must know the two immediately preceding cue locations. Reaction times typically decrease with practice on the SRT task both because the repeating sequence is being learned and because of nonspecific effects of practicing the task. Specific knowledge of the repeating sequence was assessed by changing the sequence of cue locations so that they followed a novel, balanced sequence. The increase in reaction time produced by changing the sequence indicates the amount of sequence-specific learning that has occurred. The sequences were $S_{1}(124313214234), S_{2}$ (232413143421), $N_{1}$ (343213142412), and $N_{2}$ (432423121413). Every subject received the same training or pretraining sequence $\left(S_{1}, S_{2}\right)$ to avoid introducing any additional variability in performance that might have arisen from differences in the rate at which the sequences could be learned. For one group (Memorize Young Random Pretraining), pretraining on the SRT task followed a pseudorandom sequence of 240 locations in four 60-trial blocks $\left(R_{1} R_{2} R_{3} R_{4}\right)$. This sequence of locations did not repeat, but each location and transition occurred about equally often within each block.

\section{Procedure}

Amnesic patients and six control groups received some or all of the five tasks described below (also see Table 3).

\section{Tasks}

SRT Pretraining and Training. SRT pretraining consisted of four 60-trial SRT blocks in which subjects pressed the key beneath the cue as the cue moved among the four possible locations. Pretraining was given to four control groups (Memorize Old $_{\text {, Memorize }}$ Young, Memorize $_{\text {Young }}$ Random Pretraining, and Baseline) in order to familiarize them with the SRT task and to permit the learning of nonspecific task knowledge prior to the presentation of the training sequence and prior to the test of implicit knowledge for the training sequence. For three of these groups (Memorize $\mathrm{Old}_{\text {Old }}$, Memorize Young, and Baseline), the first three blocks of pretraining contained a repeating sequence $\left(S_{2}\right)$, and the final block of pretraining introduced a novel, repeating sequence $\left(N_{2}\right)$. A significant increase in RT for this final block $\left(N_{2}\right)$ would demonstrate that subjects can express significant implicit knowledge of the pretraining sequence $\left(S_{2}\right)$. That is, an increase in RT during block $N_{2}$, compared with the preceding block of $S_{2}$, would show that nonspecific SRT learning (during the $N_{2}$ block) was not preventing the expression of implicit sequence knowledge. Moreover, in the subsequent test of implicit sequence knowledge for the training sequence $\left(S_{1}\right)$ the masking effect of nonspecific SRT skill learning could not be an explanation for a failure to observe implicit sequence knowledge.

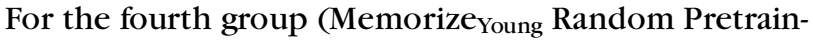
ing), pretraining consisted of four 60-trial blocks of a pseudorandom sequence. This group could acquire nonspecific task knowledge but did not learn a specific sequence during pretraining.

Two groups received SRT training (amnesic patients and CON group). SRT training consisted of practicing twenty 60-trial blocks (1200 trials). Each training block contained five repetitions of the 12-location, repeating sequence $\left(S_{1}\right)$.

Explicit Sequence Memorization Training. Subjects were instructed to watch the computer screen and attempt to memorize the sequence of locations of the target sequence $\left(S_{1}\right)$. Each cue appeared on the screen for $750 \mathrm{msec}$ with a 250 -msec intertrial interval. No keys were pressed by the subject during this task. After one presentation of the 12-location sequence, there was a short pause while subjects were instructed that they would be shown the sequence again and they should again attempt to memorize it. This procedure was repeated until subjects had observed the 12-location sequence five times. It was hypothesized that memorization of the sequence, without any SRT experience with the sequence, would lead to explicit knowledge of the training sequence without implicit sequence knowledge.

Verbal Report. After completing SRT training, subjects were asked to attempt to report the repeating sequence either verbally or by pointing in turn to the appropriate cue locations on the computer screen. Subjects were prompted to attempt to report the sequence even if they were not aware of its existence. Prompting continued until subjects reported at least eight locations (even if they had to guess). Responses were analyzed to deter- 
mine the longest section of the repeating sequence that was contained in the verbal report.

Recognition Memory Test. The recognition test was presented in the same manner as the SRT task (i.e., subjects pressed the key underneath the cue as soon as it appeared on the screen), but subjects were additionally instructed that after responding to 12 appearances of the cue, they would be asked to rate, on a 0 to 100 scale, whether they thought they had seen that same sequence of cue locations in the immediately preceding training phase of the experiment. Five different 12-item sequences were administered, and subjects made a 0 to 100 rating after each sequence. The training sequence $\left(S_{1}\right)$ was always either the second or fourth of the five sequences. The recognition test score was calculated as the rating given to the target sequence $\left(S_{1}\right)$ minus the mean rating given to the other four sequences. The recognition memory test assessed subjects explicit knowledge of the training sequence.

Implicit Test of Sequence Knowledge. Two final 60-trial blocks were given to each group in order to assess implicit sequence knowledge for the training sequence $\left(S_{1}\right)$. In the first of these blocks, a 60-trial block was given that contained five repetitions of the 12-location training sequence $\left(S_{1}\right)$. The starting position for the sequence was the same as the starting position used for SRT training and explicit sequence memorization training. The second 60-trial block consisted of five repetitions of a 12-location novel sequence $\left(N_{1}\right)$. Subjects were instructed to respond to each appearance of the asterisk by pressing the key underneath it as quickly as possible. No mention was made of any repeating sequence. Implicit knowledge of the training sequence was measured by the slower mean reaction time that occurred during the second SRT block $\left(N_{1}\right)$ compared with the first SRT block $\left(S_{1}\right)$ (i.e., the difference in mean RT between these two blocks).

\section{Subject Groups}

Amnesic Patients. The five amnesic patients received twenty 60-trial blocks of SRT training with the target sequence $S_{1}$. This training was followed by verbal report, the recognition test, and then by the implicit sequence knowledge test. The amnesic patients repeated the same procedure (with the same training sequence) in a separate session 3 to 5 months later.

CON Subjects. The CON group $(n=9)$ received the same procedure as the amnesic patients, with the two testing sessions separated by 9 to 10 months.

Memorize $_{\text {Old. }}$ The Memorize Old $_{\text {group }}(n=15)$ received four 60 -trial blocks of pretraining with the SRT task. The first three of these blocks each contained five repetitions of the 12-location, pretraining sequence $\left(S_{2}\right)$. The fourth block contained five repetitions of a novel sequence $\left(\mathrm{N}_{2}\right)$. Pretraining was followed by explicit sequence memorization training with sequence $S_{1}$, verbal report, the recognition memory test, and the implicit sequence knowledge test.

Memorize $_{\text {Young. The Memorize }}$ Young group $(n=15)$ was tested in exactly the same way as the Memorize old $_{\text {group. }}$ These subjects were younger than both the amnesic patients and the subjects in the CON and the Memorize $_{\text {old }}$ groups. For both the Memorize old $_{\text {and }}$

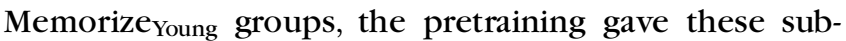
jects enough experience with the SRT task to acquire nonspecific task knowledge and enough experience to express significant knowledge of sequence $S_{2}$ (as demonstrated by slower RTs when the novel $N_{2}$ sequence was introduced). Because pretraining with repeating sequence $S_{2}$ could potentially have interfered with the ability later to express implicit knowledge of the memorized sequence $S_{1}$, two additional groups were given pretraining either with random cue locations (Memorize Young Random Pretraining) or no pretraining at

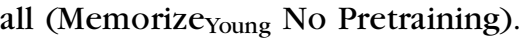

Memorize $_{\text {Young }}$ Random Pretraining. The Memorize ${ }_{\text {Young }}$ Random Pretraining group ( $n=10)$ was given SRT pretraining in which the cue followed a pseudorandom sequence of locations for four 60-trial blocks $\left(R_{1} R_{2} R_{3} R_{4}\right)$. This sequence of locations did not contain an embedded repeating sequence, but each location and transition occurred about equally often. Pretraining was followed by explicit sequence memorization training with sequence $S_{1}$, verbal report, the recognition memory test, and finally the implicit sequence knowledge test. The procedure for this group differed from the Memorize old $_{\text {and }}$

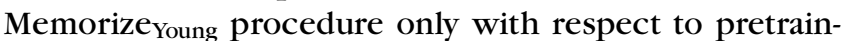
ing. Because the pretraining for the Memorize Young Random Pretraining group did not involve a repeating sequence, the pretraining should not have interfered with this group's ability to express implicit sequence knowledge of the training sequence after memorizing it.

Memorize $_{\text {Young No Pretraining. The Memorize }}$ Young No $_{\text {Nen }}$ Pretraining group $(n=10)$ was given explicit sequence memorization training with sequence $S_{1}$, verbal report, the recognition memory test, and then the implicit sequence knowledge test. The performance of this group on the implicit sequence knowledge test, in comparison with the Memorize $_{\text {Old }}$, Memorize $_{\text {Young, }}$, and Memorize ${ }_{Y o u n g}$ Random Pretraining groups, provides a way to determine whether pretraining had any effect on performing the implicit sequence knowledge test.

Baseline. The Baseline group $(n=15)$ was given the same pretraining as the Memorize Old $_{1}$ and Memorize ${ }_{Y o u n g}$ groups but was then given the implicit sequence knowledge test without any previous experience with the 
training sequence $\left(S_{1}\right)$. This group provided an estimate of baseline performance on the implicit sequence knowledge test in the absence of any opportunity to acquire either implicit or explicit knowledge of the training sequence.

\section{Acknowledgments}

This research was supported by the Medical Research Service of the Department of Veterans Affairs and by National Institute of Mental Health Grants MH24600 (Larry R. Squire) and F32 MH11150-01A1 (Paul J. Reber). We thank James Moore and Joyce Zouzounis for research assistance.

Reprint requests should be sent to Larry Squire, Veterans Affairs Medical Center 116A, 3350 La Jolla Village Drive, San Diego, CA 92161, or via e-mail: 1squire@ucsd.edu.

\section{REFERENCES}

Curran, T., \& Keele, S. (1993). Attentional and nonattentional forms of sequence learning. Journal of Experimental Psychology: Learning, Memory, and Cognition, 19, 189-202.

Dulany, D. E., Carlson, R. A., \& Dewey, G. I. (1985). A case of syntactical learning and judgment: How conscious and how abstract? Journal of Experimental Psychology: General, 113, 541-555.

Frensch, P. A., \& Miner, C. S. (1994). Effects of presentation rate and individual differences in short-term memory capacity on an indirect measure of serial learning. Memory E Cognition, 22, 96-110.

Gilbert, J., Levee, R., \& Catalano, K. (1968). A preliminary report on a new memory scale. Perceptual and Motor Skills, 27, 277-278.

Grafton, S. T., Hazeltine, E., \& Ivry, R. (1995). Functional mapping of sequence learning in normal humans. Journal of Cognitive Neuroscience, 7, 497-510.

Hamann, S. B., \& Squire, L. R. (1997). Intact perceptual memory in the absence of conscious memory. Behavioral Neuroscience, 111, 850-854.

Howard, J. H., Mutter, S. A., \& Howard, D. V. (1992). Serial pattern learning by event observation. Journal of Experimental Psychology: Learning, Memory, and Cognition, 18, 1029-1039.

Jackson, G. M., Jackson, S. R., Harrison, J., Henderson, L., \& Kennard, C. (1995). Serial reaction time learning and Parkinson's disease: Evidence for a procedural learning deficit. Neuropsychologia, 33, 577-593.

Janowsky, J. S., Shimamura, A. P., \& Squire, L. R. (1989). Source memory, impairment in patients with frontal lobe lesions. Neuropsychologia, 27, 1043-1056.

Kaplan, E. F., Goodglass, H., \& Weintraub, S. (1983). The Boston Naming Test. Philadelphia: Lea Febiger.

Knopman, D. S., \& Nissen, M. J. (1991). Procedural learning is impaired in Huntington's disease: Evidence from the serial reaction time task. Neuropsychologia, 29, 245-254.

Knowlton, B. J., Mangels, J. A., \& Squire, L. R. (1996). A neostriatal habit learning system in humans. Science, 273, 1399-1402.

Knowlton, B. J., Ramus, S. J., \& Squire, L. R. (1992). Intact artificial grammar learning in amnesia: Dissociation of classification learning and explicit memory for specific instances. Psychological Science, 3, 172-179.

Knowlton, B. J., \& Squire, L. R. (1994). The information ac- quired during artificial grammar learning. Journal of Experimental Psychology: Learning, Memory, and Cognition, 20, 79-91.

Knowlton, B. J., Squire, L. R., \& Gluck, M. (1994). Probabilistic classification learning in amnesia. Learning and Memory, 1, 106-120.

Kritchevsky, M., Squire, L. R., \& Zouzounis, J. A. (1988). Transient global amnesia: Characterization of anterograde and retrograde amnesia. Neurology, 38, 213-219.

Mattis, S. (1976). Dementia Rating Scale. In R. Bellack \& B. Keraso (Eds.), Geriatric psychiatry (pp. 77-121). New York: Grune and Stratton.

Morris, C. D., Bransford, J. D., \& Franks, J. J. (1977). Levels of processing versus transfer appropriate processing. Journal of Verbal Learning and Verbal Behavior, 16, 519-533.

Nissen, M. J., \& Bullemer, P. (1987). Attentional requirements of learning: Evidence from performance measures. Cognitive Psychology, 19, 1-32.

Nissen, M. J., Willingham, D., \& Hartman, M. (1989). Explicit and implicit remembering: When is learning preserved in amnesia? Neuropsychologia, 27, 341-352.

Osterrieth, P. A. (1944). Le test de copie d'une figure complexe [The test of copying a complex figure]. Archives de Psychologie, 30, 206-356.

Pascual-Leone, A., Grafman, J., Clark, K., Stewart, M., Massaquoi, S., Lou, J., \& Hallett, M. (1994). Procedural learning in Parkinson's disease and cerebellar degeneration. Annals of Neurology, 34, 594-602.

Pascual-Leone, A., Grafman, J., \& Hallett, M. (1994). Modulation of cortical motor output maps during development of implicit and explicit knowledge. Science, 263, 12871289.

Perruchet, P., \& Gallego, J. (1993). Association between conscious knowledge and performance in normal subjects: Reply to Cohen and Curran (1993) and Willingham, Greely, and Bardone (1993). Journal of Experimental Psychology: Learning, Memory, and Cognition, 19, 1438-1444.

Polich, J., \& Squire, L. R. (1993). P300 from amnesic patients with bilateral hippocampal lesions. EEG Clinical Neuropsychology, 86, 408-417.

Rauch, S. L., Savage, C. R., Brown, H. D., Curran, T., Alpert, N. M., Kendrick, A., Fischman, A. J., \& Kosslyn, S. M. (1995). A PET investigation of implicit and explicit sequence learning. Human Brain Mapping, 3, 271-286.

Reber, P. J., \& Squire, L. R. (1994). Parallel brain systems for learning with and without awareness. Learning and Memory, 2, 1-13.

Reed, J., \& Johnson, P. (1994). Assessing implicit learning with indirect tests: Determining what is learned about sequence structure. Journal of Experimental Psychology: Learning, Memory, and Cognition, 20, 585-594.

Roediger, H. L., III (1990). Implicit memory: Retention without remembering. American Psychologist, 45, 1043-1056.

Roediger, H. L., III, \& Blaxton, T. A. (1987). Effects of varying modality, surface features and retention interval on priming in word-fragment completion. Memory and Cognition, 15, 379-388.

Schacter, D. L. (1987). Implicit memory: History and current status. Journal of Experimental Psychology: Learning, Memory, and Cognition, 13, 501-518.

Schacter, D. L. (1990). Toward a cognitive neuropsychology of awareness: Implicit knowledge and anosognosia. Journal of Clinical and Experimental Neuropsychology, 12, 155-178.

Shanks, D. R., \& St. John, M. F. (1994a). Characteristics of dissociable human learning systems. Behavioral and Brain Sciences, 17, 367-447. 
Shanks, D. R., \& St. John, M. F. (1994b). How should implicit learning be characterized? Behavioral and Brain Sciences, 17, 427-447.

Squire, L. R. (1992). Memory and the hippocampus: A synthesis from findings with rats, monkeys, and humans. Psychological Review, 99, 195-231.

Squire, L. R., Amaral, D. G., \& Press, G. A. (1990). Magnetic resonance measurements of hippocampal formation and mammillary nuclei distinguish medial temporal lobe and diencephalic amnesia. Journal of Neuroscience, 10, 31063117.

Squire, L. R., \& Knowlton, B. J. (1995). Learning about categories in the absence of memory. Proceedings of the $\mathrm{Na}$ tional Academy of Sciences, 92, 12470-12474.

Squire, L. R., \& Shimamura, A. P. (1986). Characterizing amnesic patients for neurobehavioral study. Behavioral Neuroscience, 100, 866-877.

Squire, L. R., \& Zola, S. M. (1996). Structure and function of declarative and nondeclarative memory systems. Proceedings of the National Academy of Sciences, USA, 93, 13515-13522.

Tulving, E. (1985). How many memory systems are there? American Psychologist, 40, 385-398.

Warrington, E. K. (1984). Recognition Memory Test. Windsor, England: FER-Nelson.

Willingham, D. B., \& Koroshetz, W. J. (1993). Evidence for dissociable motor skills in Huntington's disease patients. Psychobiology, 21, 173-182.

Willingham, D. B., Nissen, M. J., \& Bullemer, P. (1989). On the development of procedural knowledge. Journal of Experimental Psychology: Learning, Memory, and Cognition, 15, 1047-1060.

Weiskrantz, L. (1991). Problems of learning and memory. One or multiple memory systems? In J. R. Krebs \& G. Horn (Eds.), Behavioral and neural aspects of learning and memory (pp. 1-10). Oxford: Clarendon. 